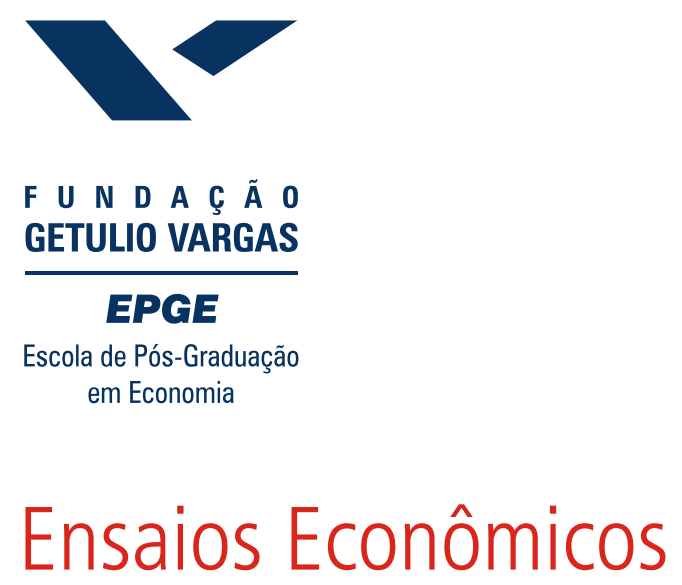

$$
\begin{aligned}
& \text { Escola de } \\
& \text { Pós Graduação } \\
& \text { em Economia } \\
& \text { da Fundação } \\
& \text { Getulio Vargas }
\end{aligned}
$$

Unbounded exchange economies with satiation: how far can we go?

Victor Filipe Martins-da-Rocha, Paulo Klinger Monteiro

Fevereiro de 2008 
Os artigos publicados são de inteira responsabilidade de seus autores. As opiniões neles emitidas não exprimem, necessariamente, o ponto de vista da Fundação Getulio Vargas. 


\title{
Unbounded exchange economies with satiation: how far can we go?
}

\author{
V. Filipe Martins-da-Rocha * Paulo K. Monteiro ${ }^{\dagger}$
}

February 28, 2008

\begin{abstract}
We unify and generalize the existence results in Werner (1987), Dana, Le Van and Magnien (1999), Allouch, Le Van and Page (2006) and Allouch and Le Van (2008). We also show that, in terms of weakening the set of assumptions, we cannot go too far.

JEL Classification: D51; C71
\end{abstract}

Keywords: Competitive equilibrium; Unbounded action sets; Satiation; Individually rational utility set; No-arbitrage

\section{Introduction}

When agents trade securities, due to the possibility of short sales, the set of portfolios is not bounded from below. This implies that the set of feasible portfolios may not be bounded and the classical existence results of Arrow and Debreu (1954) and McKenzie (1959) cannot be applied. Existence results for models with unbounded action sets have been provided by Hart (1974), Hammond (1983) and Page (1987) for security markets. In these models the utility $V^{i}(q, \theta)$ of a portfolio $\theta$ is defined by the expected utility of its return $\mathbb{E}^{\mu^{i}(q)}(r \cdot \theta)$ with respect to price-dependent beliefs $\mu^{i}(q)$. In a different context where the utility function does not depend on prices, existence results have been provided by Werner (1987), Nielsen (1989), Page and Wooders (1996), Dana et al. (1999), Page, Wooders and Monteiro (2000), Allouch, Le Van and Page (2002) and Le Van, Page and Wooders (2001). To prove existence, several conditions have been proposed to limit arbitrage opportunities. In all cases the role played by those no-arbitrage conditions was to bound the economy endogenously. Dana

\footnotetext{
${ }^{*}$ Graduate School of Economics, Getulio Vargas Foundation, Praia de Botafogo 190, 22.250900 Rio de Janeiro, Brazil. Email: victor.rocha@fgv.br

${ }^{\dagger}$ Graduate School of Economics, Getulio Vargas Foundation, Praia de Botafogo 190, 22.250900 Rio de Janeiro, Brazil. Email: paulo.klinger@fgv.br
} 
et al. (1999) proved that all these conditions imply the compactness of the individually rational utility set. ${ }_{1}^{1}$ which in turn is a sufficient condition for existence.$^{2}$

In models with bounded from below consumption sets, a crucial assumption imposed (e.g. Arrow and Debreu (1954) and McKenzie (1959)) is that agents' preferences satisfy a non-satiation property (e.g. monotonicity). Actually, what is needed is to assume that non-satiation holds only over individually feasible actions. In security markets models (like CAPM), satiation of preferences is rather a rule than an exception (see, among others, Werner (1987), Nielsen (1989), Allingham (1991), Dana et al. (1999) and Won, Hahn and Yannelis (2008)). In his seminal paper, Werner (1987) allows for satiation but he imposes that all arbitrage opportunities are uniform among agents and that each agent has a useful portfolio. His existence result was extended by Allouch et al. (2006) where a weaker non-satiation condition (ANS) is imposed: useless net trades are uniform among agents and each agent who is satiated has a non-empty set of useful net trades. Recently, a weaker non-satiation condition (WNS) was proposed by Allouch and Le Van (2008): satiation is possible provided that each agent has satiation points available to him outside the set of individually feasible consumptions. They prove that this condition is sufficient for existence of a quasi-equilibrium provided that the set of individually rational and feasible allocations is compact.

The main objective of this paper is to investigate if it is possible to unify the aforementioned existence results. There are two kinds of assumptions used to prove existence: the first one deals with satiated preferences while the second one relies on a compactness condition. Allouch et al. (2006) and Allouch and Le Van (2008) impose the weakest condition on satiation ${ }^{3}$ while Dana et al. (1999) impose the weakest compactness condition. Therefore, one may conjecture that existence is guaranteed under the two weakest conditions:

(a) the compactness condition (CU), i.e., the individually rational utility set is compact;

(b) the weak non-satiation condition (WNS), i.e., satiation is possible provided that each agent has satiation points available to him outside the set of individually feasible consumptions.

We prove that such a conjecture is not correct provided that they are more than two agents in the economy. We subsequently introduce a new condition, called strong compactness of the individually rational utility set (SCU) and we prove that it is sufficient for existence of a quasi-equilibrium when agents' preferences satisfy the weak non-satiation condition (WNS). We also show that, in

\footnotetext{
${ }^{1}$ That we call compactness condition (CU).

${ }^{2}$ Allouch (2002) introduced a weaker condition than the compactness of the individually rational utility set. In particular, an existence result was proved without assuming that preference relations are complete.

${ }^{3}$ They allow for satiation while Dana et al. (1999) do not.
} 
general, condition (SCU) is stronger than the compactness of the individually rational utility set (CU) ${ }^{4}$ but weaker than the two compactness conditions imposed in Allouch et al. (2006) and Allouch and Le Van (2008).

\section{The Model}

Consider an economy $\left(X^{i}, u^{i}, e^{i}\right)_{i \in I}$ where $I$ is a finite set, for each $i \in I$, the set $X^{i}$ is a subset of $\mathbb{R}^{J}$ with $J$ a finite set, $e^{i}$ is a vector in $\mathbb{R}^{J}$ and $u^{i}$ is a realvalued function defined on $X^{i}$. As in Werner (1987), each $j \in J$ represents a commodity which can be a consumption good as well as a financial asset. Each $i \in I$ represents an agent, $X^{i}$ his action set, $e^{i}$ his initial endowment and $u^{i}$ his utility function. Once for all the sets $\left(X^{i}\right)_{i \in I}$ and the vectors $\left(e^{i}\right)_{i \in I}$ are fixed. The economy $\left(X^{i}, u^{i}, e^{i}\right)_{i \in I}$ is then denoted by $\mathcal{E}(\boldsymbol{u})$.

We denote by $\mathrm{F}$ the set of feasible allocations, i.e., those vectors $\boldsymbol{x}=\left(x^{i}\right)_{i \in I}$ in $\boldsymbol{X}:=\prod_{i \in I} X^{i}$ satisfying

$$
\sum_{i \in I} x^{i}=\sum_{i \in I} e^{i}
$$

and by $\operatorname{Ir}(\boldsymbol{u})$ the set of individually rational allocations, i.e., those vectors $\boldsymbol{x}=$ $\left(x^{i}\right)_{i \in I}$ in $\boldsymbol{X}$ satisfying

$$
\forall i \in I, \quad u^{i}\left(x^{i}\right) \geqslant u^{i}\left(e^{i}\right) .
$$

We shall denote by $\mathrm{A}(\boldsymbol{u})$ the set $\mathrm{F} \cap \operatorname{Ir}(\boldsymbol{u})$ and by $\mathrm{A}^{i}(\boldsymbol{u})$ the projection of $\mathrm{A}(\boldsymbol{u})$ onto $X^{i}$. An action $x^{i} \in \mathrm{A}^{i}(\boldsymbol{u})$ is said individually rational and feasible. We denote by $\mathrm{A}_{c}^{i}(\boldsymbol{u})$ the set $X^{i} \backslash \mathrm{A}^{i}(\boldsymbol{u})$ the set of actions $x^{i} \in X^{i}$ that are not individually rational and feasible. We let $\mathcal{U}(\boldsymbol{u})$ denote the utility set defined by

$$
\mathcal{U}(\boldsymbol{u})=\left\{\boldsymbol{\lambda} \in \mathbb{R}^{I}: \exists \boldsymbol{x} \in \mathrm{F} \cap \operatorname{Ir}(\boldsymbol{u}), \quad \forall i \in I, \quad u^{i}\left(e^{i}\right) \leqslant \lambda^{i} \leqslant u^{i}\left(x^{i}\right)\right\} .
$$

From now on we assume that the economy $\left(X^{i}, u^{i}, e^{i}\right)_{i \in I}$ satisfies the following list of standard conditions:

(A.1) the set $X^{i}$ is closed convex containing $e^{i}$;

(A.2) the function $u^{i}$ is upper semi-continuous and strictly quasi-concave. $5^{5}$

\section{Existence under a non-satiation condition (NS)}

If $x^{i} \in X^{i}$ is such that the set $P^{i}\left(x^{i}\right):=\left\{y^{i} \in X^{i}: u^{i}\left(y^{i}\right)>u^{i}\left(x^{i}\right)\right\}$ is empty, then $x^{i}$ is called a satiation point of $u^{i}$. We shall denote by $S^{i}\left(u^{i}\right)$ the set of

\footnotetext{
${ }^{4}$ Except if there are at most two agents in the economy. In that case, both conditions (SCU) and (CU) coincide.

${ }^{5}$ The function $u^{i}$ is upper semi-continuous if for each $c \in \mathbb{R}$, the upper level set $\{x \in$ $\left.X^{i}: u^{i}(x) \geqslant c\right\}$ is closed in $X^{i}$. The function $u^{i}$ is strictly quasi-concave if for every $x$ and $y$ in $X^{i}$, if $u^{i}(y)>u^{i}(x)$, then $u^{i}(\lambda y+(1-\lambda) x)>u^{i}(x)$ for every $\lambda \in(0,1]$.
} 
satiation points of $u^{i}$ on $X^{i}$, i.e.

$$
S^{i}\left(u^{i}\right):=\operatorname{argmax}\left\{u^{i}\left(x^{i}\right): x^{i} \in X^{i}\right\} .
$$

When there is no satiation point in $X^{i}$, the set $S^{i}\left(u^{i}\right)$ is empty. We recall now the definition of a quasi-equilibrium.

Definition 3.1. Given an economy $\mathcal{E}(\boldsymbol{u})$, a couple $(p, \boldsymbol{x})$ where $0 \neq p \in \mathbb{R}^{J}$ and $\boldsymbol{x}=\left(x^{i}\right)_{i \in I}$ is a feasible allocation in $\mathrm{F}$, is a quasi-equilibrium of $\mathcal{E}(\boldsymbol{u})$ if for each $i$

(a) the action $x^{i}$ satisfies the budget restriction $p \cdot x^{i} \leqslant p \cdot e^{i}$;

(b) the action $x^{i}$ is weakly optimal in the budget set in the sense that for each $y^{i} \in P^{i}\left(x^{i}\right)$, we have $p \cdot y^{i} \geqslant p \cdot e^{i}$.

If the individually rational utility $\operatorname{set} \mathcal{U}(\boldsymbol{u})$ is compact then a sufficient condition for existence of a quasi-equilibrium is the following non-satiation condition:

(NS) the set $S^{i}\left(u^{i}\right) \cap \mathrm{A}^{i}(\boldsymbol{u})$ is empty for each $i$.

Theorem 3.1 (Dana, Le Van and Magnien). If the two following conditions are satisfied

(a.1) the individually rational utility $\operatorname{set} \mathcal{U}(\boldsymbol{u})$ is compact,

(b.2) the non-satiation condition (NS) is satisfied,

then there exists a quasi-equilibrium.

The proof of Theorem 3.1 follows from standard arguments: see e.g. Dana et al. (1999). Observe that if the set $\mathrm{A}(\boldsymbol{u})=\mathrm{F} \cap \operatorname{Ir}(\boldsymbol{u})$ of feasible and individually rational allocations is compact then the individually rational utility set $\mathcal{U}(\boldsymbol{u})$ is trivially compact. However, the converse is not in general true.

\section{A weak non-satiation condition (WNS)}

Recently, Allouch and Le Van (2008) introduced a weaker non-satiation condition:

(WNS) for every individually rational and feasible action $x^{i} \in \mathrm{A}^{i}(\boldsymbol{u})$, there exists a action $y^{i} \in \mathrm{A}_{c}^{i}(\boldsymbol{u})$ which is not individually rational and feasible but satisfies $u^{i}\left(y^{i}\right) \geqslant u^{i}\left(x^{i}\right)$.

Condition (WNS) is obviously weaker than the non-satiation condition (NS). The weak non-satiation condition (WNS) is satisfied if and only if,

$$
\forall i \in I, \quad S^{i}\left(u^{i}\right) \cap \mathrm{A}^{i}(\boldsymbol{u}) \neq \emptyset \Longrightarrow S^{i}\left(u^{i}\right) \cap \mathrm{A}_{c}^{i}(\boldsymbol{u}) \neq \emptyset .
$$


In other words, under condition (WNS), each agent $i$ may have satiation points that are individually rational and feasible, but the set of satiation points must be large enough such that there exists satiation actions which are not individually rational and feasible.

Allouch and Le Van (2008) proved ${ }^{6}$ that if the set $\mathrm{A}(\boldsymbol{u})=\mathrm{F} \cap \operatorname{Ir}(\boldsymbol{u})$ of feasible and individually rational allocations is compact, then the weak non-satiation condition (WNS) is sufficient for existence.

Theorem 4.1 (Allouch and Le Van). If the two following conditions are satisfied

(a.3) the set $\mathrm{A}(\boldsymbol{u})=\mathrm{F} \cap \operatorname{Ir}(\boldsymbol{u})$ of feasible and individually rational allocations is compact,

(b.1) the the weak non-satiation condition (WNS) is satisfied,

then there exists a quasi-equilibrium.

This result is not comparable with the one by Dana et al. (1999). Indeed, Allouch and Le Van (2008) consider a weaker non-satiation condition but a stronger compactness assumption. A natural question is whether an existence result generalizing both results of Dana et al. (1999) and Allouch and Le Van (2008) is possible. One may conjecture that the weakest conditions of both results are sufficient for existence.

Conjecture 4.1. If the two following conditions are satisfied

(a.1) the individually rational utility $\operatorname{set} \mathcal{U}(\boldsymbol{u})$ is compact,

(b.1) the weak non-satiation condition (WNS) is satisfied,

then there exists a quasi-equilibrium.

Our first result of the paper is to prove that Conjecture 4.1 is not correct. We provide a counterexample in the following section. In Section 6 we prove that Conjecture 4.1 is correct if there is at most two agents in the economy.

\section{Conjecture 4.1 is false}

In this section we consider an economy with three agents and two commodities such that the individually rational utility set is compact and the weak nonsatiation condition is satisfied, but for which there is no quasi-equilibrium.

We pose $I=\left\{i_{1}, i_{2}, i_{3}\right\}$ and $J=\left\{j_{1}, j_{2}\right\}$. The action set of agent $i_{1}$ is given by

$$
X^{i_{1}}=[-1, \infty) \times[-1 / 2, \infty) ;
$$

\footnotetext{
${ }^{6}$ We propose in Appendix A an alternative proof based on a very general existence result by Florenzano (2003).
} 
his utility function is given by

$$
u^{i_{1}}(x)=\left\{\begin{array}{ccc}
\frac{x_{j_{2}}+x_{j_{1}}}{x_{j_{2}}+1} & \text { if } & -1 \leqslant x_{j_{1}} \leqslant 1 ; \\
x_{j_{1}} & \text { if } & 1 \leqslant x_{j_{1}},
\end{array}\right.
$$

and he has no initial endowment, i.e., $e^{i_{1}}=0$. The action set of agent $i_{2}$ is given by

$$
X^{i_{2}}=[-1,2] \times \mathbb{R}
$$

his utility function is given by

$$
u^{i_{2}}(x)=x_{j_{1}},
$$

and he has no initial endowment, i.e., $e^{i_{2}}=0$. The action set of agent $i_{3}$ is given by

$$
X^{i_{3}}=[-3, \infty) \times\{0\} ;
$$

his utility function is given by

$$
u^{i_{3}}(x)=0,
$$

and he has no initial endowment, i.e., $e^{i_{3}}=0$.

Proposition 5.1. The economy satisfies Assumptions (A.1) and (A.2). Moreover, the individually rational utility set $\mathcal{U}(\boldsymbol{u})$ is compact and the weak non-satiation condition (WNS) is satisfied.

Proof. It is immediate that Assumptions (A.1) and (A.2) are satisfied. We propose to prove that the utility set $\mathcal{U}(\boldsymbol{u})$ is bounded and closed. Let $\left(\lambda^{i_{1}}, \lambda^{i_{2}}, \lambda^{i_{3}}\right)$ in $\mathcal{U}(\boldsymbol{u})$, then there exists $\boldsymbol{x} \in \mathrm{F}$ such that

$$
\forall i \in I, \quad 0 \leqslant \lambda^{i} \leqslant u^{i}\left(x^{i}\right) .
$$

This implies that $\lambda^{i_{2}} \in[0,2]$ and $\lambda^{i_{3}}=0$. Moreover, since $x^{i_{1}}=-x^{i_{2}}-x^{i_{3}}$, it follows that $x^{i_{1}} \leqslant 4$ and $\lambda^{i_{1}} \leqslant 4$. As a consequence

$$
\mathcal{U}(\boldsymbol{u}) \subset[0,4] \times[0,2] \times\{0\}
$$

is a bounded set. In order to prove that $\mathcal{U}(\boldsymbol{u})$ is closed, consider a sequence $\boldsymbol{\lambda}_{n}$ in $\mathcal{U}(\boldsymbol{u})$ converging to some $\lambda$.

If $\lambda^{i_{1}} \leqslant 1$ then

$$
\forall i \in I, \quad 0 \leqslant \lambda^{i} \leqslant u^{i}\left(y^{i}\right)
$$

where 7

$$
y^{i_{1}}=\mathbf{1}_{j_{1}}, \quad y^{i_{2}}=2 \mathbf{1}_{j_{1}} \quad \text { and } \quad y^{i_{3}}=-3 \mathbf{1}_{j_{1}} .
$$

Since $\boldsymbol{y}$ belongs to $\mathrm{A}(\boldsymbol{u})$ it follows that $\boldsymbol{\lambda}$ belongs to $\mathcal{U}(\boldsymbol{u})$.

\footnotetext{
${ }^{7}$ We denote by $\mathbf{1}_{j_{1}}$ the vector $z \in \mathbb{R}^{j}$ such that $z_{j_{1}}=1$ and $z_{j_{2}}=0$.
} 
Assume now that $\lambda^{i_{1}}>1$. Since $\boldsymbol{\lambda}_{n}$ belongs to $\mathcal{U}(\boldsymbol{u})$, there exists a sequence $\boldsymbol{x}_{n}$ in $\mathrm{A}(\boldsymbol{u})$ such that

$$
\forall i \in I, \quad 0 \leqslant \lambda_{n}^{i} \leqslant u^{i}\left(x_{n}^{i}\right)
$$

For each $n$, we have

$$
x_{n}^{i_{1}}+x_{n}^{i_{2}}+x_{n}^{i_{3}}=0 \quad \text { and } \quad\left(x_{j_{1}, n}^{i_{1}}, x_{j_{1}, n}^{i_{2}}, x_{j_{3}, n}^{i_{3}}\right) \geqslant(-1,-1,-3) .
$$

Passing to a subsequence if necessary, we can suppose that the sequence

$$
\left(x_{j_{1}, n}^{i_{1}}, x_{j_{1}, n}^{i_{2}}, x_{j_{3}, n}^{i_{3}}\right)_{n}
$$

converges to a vector $\left(x_{j_{1}}^{i_{1}}, x_{j_{1}}^{i_{2}}, x_{j_{1}}^{i_{3}}\right)$ in $\mathbb{R}^{3}$ satisfying

$$
x_{j_{1}}^{i_{1}}+x_{j_{1}}^{i_{2}}+x_{j_{1}}^{i_{3}}=0 .
$$

Since $\lambda^{i_{1}}>1$, for $n$ large enough we have $x_{j_{1}, n}^{i_{1}}>1$, implying that $u^{i_{1}}\left(x_{n}^{i_{1}}\right)=$ $x_{j_{1}, n}^{i_{1}}$. It follows that for $n$ large enough

$$
\lambda_{n}^{i_{1}} \leqslant x_{j_{1}, n}^{i_{1}}, \quad \lambda_{n}^{i_{2}} \leqslant x_{j_{1}, n}^{i_{2}} \quad \text { and } \quad \lambda_{n}^{i_{3}}=0 .
$$

Passing to the limit, we get

$$
\lambda^{i_{1}} \leqslant x_{j_{1}}^{i_{1}}, \quad \lambda^{i_{2}} \leqslant x_{j_{1}}^{i_{2}} \quad \text { and } \quad \lambda^{i_{3}}=0 .
$$

This implies that

$$
\forall i \in I, \quad 0 \leqslant \lambda^{i} \leqslant u^{i}\left(z^{i}\right)
$$

where

$$
z^{i_{1}}=x_{j_{1}}^{i_{1}} \mathbf{1}_{j_{1}}, \quad z^{i_{2}}=x_{j_{1}}^{i_{2}} \mathbf{1}_{j_{1}} \quad \text { and } \quad z^{i_{3}}=x_{j_{1}}^{i_{3}} \mathbf{1}_{j_{1}} .
$$

Since $z$ belongs to $\mathrm{A}(\boldsymbol{u})$ it follows that $\boldsymbol{\lambda}$ belongs to $\mathcal{U}(\boldsymbol{u})$.

Agent $i_{1}$ satisfies the non-satiation Assumption (NS). Agents $i_{2}$ and $i_{3}$ satisfy Assumption (WNS). Indeed, if $\boldsymbol{x}$ belongs to F then

$$
\left(x_{j_{1}}^{i_{1}}, x_{j_{1}}^{i_{2}}, x_{j_{1}}^{i_{3}}\right) \in[-1,4] \times[-1,4] \times[-3,2] .
$$

implying that the action $3 \mathbf{1}_{j_{1}}$ belongs to $S^{i_{3}}\left(u^{i_{3}}\right) \cap \mathrm{A}_{c}^{i_{3}}(\boldsymbol{u})$. Now since $\boldsymbol{x} \in \boldsymbol{X}$, we have

$$
x^{i_{1}} \geqslant-1 \text { and } x^{i_{3}}=0
$$

implying by feasibility that $x^{i_{2}} \leqslant 1$. It follows that the vector $2 \mathbf{1}_{j_{1}}+2 \mathbf{1}_{j_{2}}$ belongs to $S^{i_{2}}\left(u^{i_{2}}\right) \cap \mathrm{A}_{c}^{i_{2}}(\boldsymbol{u})$.

Remark 5.1. Consider the sequence $\left(\boldsymbol{x}_{n}\right)_{n}$ of feasible allocations defined by

$$
x_{n}^{i_{1}}=-\mathbf{1}_{j_{1}}+n \mathbf{1}_{j_{2}}, \quad x_{n}^{i_{2}}=\mathbf{1}_{j_{1}}-n \mathbf{1}_{j_{2}} \quad \text { and } \quad x_{n}^{i_{3}}=0 .
$$


For each $n$, we let $\lambda_{n}^{i}:=u^{i}\left(x_{n}^{i}\right)$. The sequence $\left(\boldsymbol{\lambda}_{n}\right)_{n}$ belongs to the utility set and

$$
\lim _{n \rightarrow \infty}\left(\lambda_{n}^{i_{1}}, \lambda_{n}^{i_{2}}, \lambda_{n}^{i_{3}}\right)=(1,1,0) .
$$

The limit $\boldsymbol{\lambda}:=(1,1,0)$ belongs to the utility set since $u^{i}\left(x^{i}\right)=\lambda^{i}$ with

$$
x^{i_{1}}=\mathbf{1}_{j_{1}}, \quad x^{i_{2}}=\mathbf{1}_{j_{1}} \quad \text { and } \quad x^{i_{3}}=-2 \mathbf{1}_{j_{1}} .
$$

The role of third agent to ensure the compactness of the utility set is crucial in this example. Indeed, if we consider the same economy but with only agents $i_{1}$ and $i_{2}$, then for each $n$ the pair $\left(\lambda_{n}^{i_{1}}, \lambda_{n}^{i_{2}}\right)$ belongs to the utility set but the limit $(1,1)$ does not.

Proposition 5.2. There is no quasi-equilibrium.

Proof. Assume that $(p, \boldsymbol{x})$ is a quasi-equilibrium. For each $i$, we have $p \cdot x^{i} \leqslant$ $p \cdot e^{i}=0$. Since the allocation $\boldsymbol{x}$ is feasible, it follows that $p \cdot x^{i}=0$, in other words the vector $x^{i}$ belongs to the budget line $L(p)$ defined by

$$
L(p):=\left\{x \in \mathbb{R}^{J}: p \cdot x=p_{j_{1}} x_{j_{1}}+p_{j_{2}} x_{j_{2}}=0\right\} .
$$

Case 1: Assume $x_{j_{1}}^{i_{2}}<2$. For every $y \in X^{i_{2}}$ with $y_{j_{1}}>0$, we have $x^{i_{2}}+$ $\alpha y \in P^{i_{2}}\left(x^{i_{2}}\right)$ for $\alpha>0$ small enough. This implies that $p \cdot y \geqslant 0$. Then necessarily $p_{j_{2}}=0$ and $p_{j_{1}}>0$. This implies that $L(p)=\left\{x \in \mathbb{R}^{J}: x_{j_{1}}=\right.$ $0\}$. Therefore $x_{j_{1}}^{i_{1}}=0$. But, for $\alpha>0$ small enough, we have $x^{i_{1}}+\mathbf{1}_{j_{2}}-$ $\alpha \mathbf{1}_{j_{1}} \in P^{i_{1}}\left(x^{i_{1}}\right)$ implying by weak optimality that $p_{j_{1}}(-\alpha)+p_{j_{2}} \geqslant 0$. This contradicts the fact that $p_{j_{2}}=0$ and $p_{j_{1}}>0$.

Case 2.1: Assume $x_{j_{1}}^{i_{2}}=2$ and $x_{j_{2}}^{i_{2}} \neq 0$. Since $x^{i_{2}}$ belongs to the budget line $L(p)$ it follows that $p_{j_{1}} \neq 0$. Then the only possibility for $x^{i_{3}}$ to belong to $L(p) \cap X^{i_{3}}$ is that $x^{i_{3}}=0$. It then follows that $x_{j_{1}}^{i_{1}}=-2$ which yields a contradiction with the fact that $x^{i_{1}} \in X^{i_{1}}$.

Case 2.2: Assume $x_{j_{1}}^{i_{2}}=2$ and $x_{j_{2}}^{i_{2}}=0$. Since $x^{i_{2}}$ belongs to the budget line $L(p)$ it follows that $p_{j_{1}}=0$. As a consequence we must have $x_{j_{2}}^{i_{1}}=0$. But, for $\alpha>0$ small enough, we have $x^{i_{1}}+\mathbf{1}_{j_{1}}-\alpha \mathbf{1}_{j_{2}} \in P^{i_{1}}\left(x^{i_{1}}\right)$ implying by weak optimality that $p_{j_{1}}+p_{j_{2}}(-\alpha) \geqslant 0$. Since $p_{j_{1}}=0$ this implies $p_{j_{2}}<0$. Similarly, for $\alpha>0$ small enough, we have $x^{i_{1}}-\mathbf{1}_{j_{1}}+\alpha \mathbf{1}_{j_{2}} \in P^{i_{1}}\left(x^{i_{1}}\right)$ implying by weak optimality that $-p_{j_{1}}+p_{j_{2}}(\alpha) \geqslant 0$. This contradicts the fact that $p_{j_{1}}=0$ and $p_{j_{2}}<0$. 


\section{General existence result under Assumption (WNS)}

Keeping the generality of the non-satiation condition introduced by Allouch and Le Van (2008), we propose to investigate under which additional conditions Conjecture 4.1 is correct. Actually, we introduce a condition called strong compactness of the utility set (SCU) under which Conjecture 4.1 is correct.

Definition 6.1. The individually rational utility set $\mathcal{U}(\boldsymbol{u})$ is strongly compact if

(SCU) for every sequence $\left(\boldsymbol{x}_{n}\right)_{n}$ in $\mathrm{F} \cap \operatorname{Ir}(\boldsymbol{u})$ of feasible and individually rational allocations there exist a feasible allocation $\boldsymbol{y}$ and a subsequence $\left(\boldsymbol{x}_{n_{k}}\right)_{k}$ satisfying

$$
\forall i \in I, \quad u^{i}\left(y^{i}\right) \geqslant \lim _{k \rightarrow \infty} u^{i}\left(x_{n_{k}}^{i}\right)
$$

together with 8

$$
\forall i \in I, \quad \lim _{k \rightarrow \infty} \frac{\mathbf{1}_{S^{i}\left(u^{i}\right)}\left(x_{n_{k}}^{i}\right)}{1+\left\|x_{n_{k}}^{i}\right\|^{2}}\left(y^{i}-x_{n_{k}}^{i}\right)=0
$$

Remark 6.1. It is straightforward to check that the strong compactness of the individually rational utility implies its compactness. Indeed, assume that $\mathcal{U}(\boldsymbol{u})$ is strongly compact and let $\left(\boldsymbol{\lambda}_{n}\right)_{n}$ be sequence in $\mathcal{U}(\boldsymbol{u})$. There exists a sequence $\left(\boldsymbol{x}_{n}\right)_{n}$ in $\mathrm{F} \cap \operatorname{Ir}(\boldsymbol{u})$ of feasible and individually rational allocations satisfying $u^{i}\left(x_{n}^{i}\right) \geqslant \lambda_{n}^{i} \geqslant u^{i}\left(e^{i}\right)$. From (SCU), there exists a feasible allocation $\boldsymbol{y}$ and a subsequence $\left(\boldsymbol{x}_{n_{k}}\right)_{k}$ satisfying

$$
\forall i \in I, \quad u^{i}\left(y^{i}\right) \geqslant \lim _{k \rightarrow \infty} u^{i}\left(x_{n_{k}}^{i}\right) .
$$

This implies that for each $i$ the subsequence $\left(\lambda_{n_{k}}^{i}\right)_{k}$ is bounded. Passing to another subsequence if necessary, we can assume without any loss of generality that the sequence $\left(\lambda_{n_{k}}^{i}\right)_{k}$ converges to some $\lambda^{i}$. From (6.3) it follows that $\boldsymbol{\lambda}$ belongs to $\mathcal{U}(\boldsymbol{u})$.

Remark 6.2. Observe that if $\left(\boldsymbol{x}_{n}\right)_{n}$ in $\mathrm{F} \cap \operatorname{Ir}(\boldsymbol{u})$ then for each $i$ we have

$$
\mathbf{1}_{S^{i}\left(u^{i}\right)}\left(x_{n}^{i}\right)=\mathbf{1}_{S^{i}\left(u^{i}\right) \cap A^{i}(\boldsymbol{u})}\left(x_{n}^{i}\right) .
$$

This implies that under the usual non-satiation Assumption (NS), the individually rational utility set $\mathcal{U}(\boldsymbol{u})$ is strongly compact if and only if it is compact.

Remark 6.3. Passing to subsequences, it is possible to prove that the strong compactness of individually rational utility set $\mathcal{U}(\boldsymbol{u})$ is equivalent to the following statement: For every sequence $\left(\boldsymbol{x}_{n}\right)_{n}$ in $\mathrm{F} \cap \operatorname{Ir}(\boldsymbol{u})$ of feasible and individually

\footnotetext{
${ }^{8}$ If $A$ is a subset of $\mathbb{R}^{J}$ then $\mathbf{1}_{A}$ is the function from $\mathbb{R}^{J}$ to $\{0,1\}$ defined by $\mathbf{1}_{A}(x)=1$ if $x \in A$ and $\mathbf{1}_{A}(x)=0$ elsewhere.
} 
rational allocations there exist a feasible allocation $\boldsymbol{y}$ and a subsequence $\left(\boldsymbol{x}_{n_{k}}\right)_{k}$ satisfying

$$
\forall i \in I, \quad u^{i}\left(y^{i}\right) \geqslant \lim _{k \rightarrow \infty} u^{i}\left(x_{n_{k}}^{i}\right)
$$

and such that $I=I_{s} \cup I_{c} \cup I_{u}$ where

$$
I_{s}=\left\{i \in I: x_{n_{k}}^{i} \notin S^{i}\left(u^{i}\right), \forall k \in \mathbb{N}\right\}, I_{c}:=\left\{i \in I \backslash I_{s}: \lim _{k \rightarrow \infty} x_{n_{k}}^{i}=y^{i}\right\}
$$

and

$$
I_{u}=\left\{i \in I \backslash I_{s}: \lim _{k \rightarrow \infty}\left\|x_{n_{k}}^{i}\right\|=\infty\right\} .
$$

The main existence result of this paper is the following generalization of Theorem 3 in Dana et al. (1999) (see Theorem 3.1) and Theorem 2 in Allouch and Le Van (2008).

Consider a strongly standard economy $\mathcal{E}(\boldsymbol{u})$ satisfying Assumption (WNS), then there exists a quasi-equilibrium.

Theorem 6.1. If the two following conditions are satisfied

(a.2) the individually rational utility $\operatorname{set} \mathcal{U}(\boldsymbol{u})$ is strongly compact,

(b.1) the weak non-satiation condition (WNS) is satisfied, then there exists a quasi-equilibrium.

Proof of Theorem 6.1. Let $\mathcal{E}(\boldsymbol{u})$ be an economy satisfying the weak non-satiation condition (WNS) and such that the individually rational utility set $\mathcal{U}(\boldsymbol{u})$ is strongly compact.

Lemma 6.1. For each agent $i$, the set

$$
\operatorname{argmax}\left\{u^{i}(x): x \in \mathrm{A}^{i}(\boldsymbol{u})\right\}
$$

is non-empty.

Proof of Lemma 6.1 There exists a sequence $\left(x_{n}^{i}\right)_{n}$ in $A^{i}(\boldsymbol{u})$ such that

$$
\lim _{n \rightarrow \infty} u^{i}\left(x_{n}^{i}\right)=\sup u^{i}\left(A^{i}(\boldsymbol{u})\right) .
$$

Since $x_{n}^{i}$ belongs to $A^{i}(\boldsymbol{u})$ there exists $\left(x_{n}^{k}\right)_{k \neq i} \in \prod_{k \neq i} X^{k}$ such that $\left(x_{n}^{k}\right)_{k \in I}$ belongs to $\mathrm{A}(\boldsymbol{u})$. For each $n$ the family $\left(u^{k}\left(x_{n}^{k}\right)\right)_{k \in I}$ belongs to $\mathcal{U}(\boldsymbol{u})$. It follows from Assumption (A.3) that there exists $\boldsymbol{\xi} \in \mathrm{A}(\boldsymbol{u})$ such that (passing to a subsequence if necessary)

$$
\forall k \in I, \quad u^{k}\left(\xi^{k}\right) \geqslant \lim _{n \rightarrow \infty} u^{k}\left(x_{n}^{k}\right) .
$$

It follows that $\xi^{i} \in \mathrm{A}^{i}(\boldsymbol{u})$ and $u^{i}\left(\xi^{i}\right) \geqslant \sup u^{i}\left(\mathrm{~A}^{i}(\boldsymbol{u})\right)$, in other words

$$
\xi^{i} \in \operatorname{argmax}\left\{u^{i}(x): x \in \mathrm{A}^{i}(\boldsymbol{u})\right\} .
$$


Let $\xi^{i}$ be an element of $\operatorname{argmax}\left\{u^{i}(x): x \in \mathrm{A}^{i}(\boldsymbol{u})\right\}$. Applying Assumption (WNS), there exists $\zeta^{i} \in \mathrm{A}_{c}^{i}(\boldsymbol{u})$ such that $u^{i}\left(\zeta^{i}\right) \geqslant u^{i}\left(\xi^{i}\right)$.

For each $i$, we let $v^{i}$ be the function defined on $X^{i}$ by

$$
\forall x \in X^{i}, \quad v^{i}(x)=u^{i}(x)+\mathbf{1}_{S^{i}\left(u^{i}\right)}(x) \exp \left\{-d\left(x, \zeta^{i}\right)\right\}
$$

where $\mathbf{1}_{S^{i}\left(u^{i}\right)}$ is the indicator function of the set $S^{i}\left(u^{i}\right)$. Observe that if the economy $\mathcal{E}(u)$ satisfies the non-satiation assumption (NS) then, for each agent $i$, we have $v^{i}=u^{i}$. We claim that the economy $\mathcal{E}(\boldsymbol{v})$ satisfies all the conditions required to apply Theorem 3.1 .

Claim 6.1. The economy $\mathcal{E}(\boldsymbol{v})$ is standard.

Proof of Claim 6.1. We only have to prove that Assumptions (A.2) and (A.3) are satisfied. We denote by $M^{i}$ the extended real number defined by

$$
M^{i}=\sup \left\{u^{i}(x): x \in X^{i}\right\} .
$$

Let $c \in \mathbb{R} \cup\{\infty\}$ then

$$
\left\{x \in X^{i}: v^{i}(x) \geqslant c\right\}= \begin{cases}S^{i}\left(u^{i}\right) \cap B\left(\zeta^{i},-\ln \left(c-M^{i}\right)\right) & \text { if } c \geqslant M^{i} \\ \left\{x \in X^{i}: u^{i}(x) \geqslant c\right\} & \text { if } c<M^{i}\end{cases}
$$

where $B\left(\zeta^{i}, r\right)=\left\{x \in \mathbb{R}^{j}: d\left(\zeta^{i}, x\right) \leqslant r\right\}$ if $r \geqslant 0$ and $B\left(\zeta^{i}, r\right)=\emptyset$ if $r<0$. It follows that the set $\left\{x \in X^{i}: v^{i}(x) \geqslant c\right\}$ is closed convex, implying that the economy $\mathcal{E}(\boldsymbol{v})$ satisfies Assumption (A.2).

We prove now that the utility set $\mathcal{U}(\boldsymbol{v})$ is compact. Let $\left(\boldsymbol{\lambda}_{n}\right)_{n}$ be a sequence in $\mathcal{U}(\boldsymbol{v})$, there exists a sequence $\left(\boldsymbol{x}_{n}\right)_{n}$ of allocations in $\mathrm{F} \cap \operatorname{Ir}(\boldsymbol{v})$ such that for each $n$

$$
\forall i \in I, \quad v^{i}\left(e^{i}\right) \leqslant \lambda_{n}^{i} \leqslant v^{i}\left(x_{n}^{i}\right) .
$$

Since $v^{i}\left(e^{i}\right) \geqslant u^{i}\left(e^{i}\right)$ the allocation $\boldsymbol{x}_{n}$ also belongs to $\mathrm{F} \cap \operatorname{Ir}(\boldsymbol{u})$. The individually rational utility set $\mathcal{U}(\boldsymbol{u})$ is strongly compact, therefore, passing to a subsequence if necessary, we may assume that there exists an allocation $\boldsymbol{y}$ in $\mathrm{F} \cap \operatorname{Ir}(\boldsymbol{u})$ satisfying

$$
\forall i \in I, \quad u^{i}\left(y^{i}\right) \geqslant \lim _{n} u^{i}\left(x_{n}^{i}\right) .
$$

and such that $I=I_{s} \cup I_{c} \cup I_{u}$ where

$$
I_{s}=\left\{i \in I: x_{n}^{i} \notin S^{i}\left(u^{i}\right), \forall n \in \mathbb{N}\right\}, \quad I_{c}:=\left\{i \in I \backslash I_{s}: \lim _{n \rightarrow \infty} x_{n}^{i}=y^{i}\right\}
$$

and

$$
I_{u}=\left\{i \in I \backslash I_{s}: \lim _{n \rightarrow \infty}\left\|x_{n}^{i}\right\|=\infty\right\} .
$$

Claim 6.2. For each $i$ we have

$$
\lim _{n \rightarrow \infty} v^{i}\left(x_{n}^{i}\right) \leqslant v^{i}\left(y^{i}\right)
$$


Proof of Claim 6.2. Let $i \in I_{s}$, by construction of $v^{i}$ we have $v^{i}\left(x_{n}^{i}\right)=u^{i}\left(x_{n}^{i}\right)$ for all $n$. It follows that

$$
\lim _{n \rightarrow \infty} v^{i}\left(x_{n}^{i}\right)=\lim _{n \rightarrow \infty} u^{i}\left(x_{n}^{i}\right) \leqslant u^{i}\left(y^{i}\right) .
$$

The desired result follows from the fact that $u^{i}\left(y^{i}\right) \leqslant v^{i}\left(y^{i}\right)$.

For each $i \in I_{c}$ the result follows from convergence of sequence $\left(x_{n}^{i}\right)_{n}$ to $y^{i}$ and the upper semicontinuity of $v^{i}$.

Now let $i \in I_{u}$, since

$$
\lim _{n \rightarrow \infty} d\left(x_{n}^{i}, \zeta^{i}\right)=+\infty
$$

we have

$$
\lim _{n \rightarrow \infty} v^{i}\left(x_{n}^{i}\right)=\lim _{n \rightarrow \infty} u^{i}\left(x_{n}^{i}\right) .
$$

The desired result follows from

$$
\lim _{n \rightarrow \infty} u^{i}\left(x_{n}^{i}\right) \leqslant u^{i}\left(y^{i}\right) \leqslant v^{i}\left(y^{i}\right) .
$$

Combining (6.5) and Claim 6.2 we prove that the set $\mathcal{U}(\boldsymbol{v})$ is compact.

In order to apply Theorem 3.1, it is sufficient to prove that the non-satiation condition (NS) is satisfied. This follows from the construction of the function $v^{i}$. Indeed, let $x^{i}$ be a vector in $A^{i}(\boldsymbol{v})$. If there exists $y^{i} \in X^{i}$ satisfying $u^{i}\left(y^{i}\right)>$ $u^{i}\left(x^{i}\right)$ then $x^{i}$ does not belong to $S^{i}\left(u^{i}\right)$ and

$$
v^{i}\left(y^{i}\right) \geqslant u^{i}\left(y^{i}\right)>u^{i}\left(x^{i}\right)=v^{i}\left(x^{i}\right)
$$

implying that $x^{i}$ does not belong to $S^{i}\left(v^{i}\right)$. Now assume that $x^{i}$ belongs to $S^{i}\left(u^{i}\right)$. There exists $\left(x^{k}\right)_{k \neq i}$ such that $\left(x^{k}\right)_{k \in I}$ belongs to $A(\boldsymbol{v})=\mathrm{F} \cap \operatorname{Ir}(\boldsymbol{v})$. Since for each $v^{i}\left(e^{i}\right) \geqslant u^{i}\left(e^{i}\right)$, we have that $\left(x^{k}\right)_{k \in I}$ also belongs to $A(\boldsymbol{u})=\mathrm{F} \cap \operatorname{Ir}(\boldsymbol{u})$ and therefore $x^{i}$ belongs to $A^{i}(\boldsymbol{u})$. By construction the vector $\zeta^{i}$ does not belong to $A^{i}(\boldsymbol{u})$, implying that

$$
\begin{aligned}
v^{i}\left(\zeta^{i}\right) & =u^{i}\left(\zeta^{i}\right)+\mathbf{1}_{S^{i}\left(u^{i}\right)}\left(\zeta^{i}\right) \exp \{0\} \\
& \geqslant u^{i}\left(x^{i}\right)+1 \\
& >u^{i}\left(x^{i}\right)+\exp \left\{-d\left(x^{i}, \zeta^{i}\right)\right\}=v^{i}\left(x^{i}\right) .
\end{aligned}
$$

Therefore the action $x^{i}$ does not belong to $S^{i}\left(v^{i}\right)$. We have thus proved that

$$
A^{i}(\boldsymbol{v}) \cap S^{i}\left(v^{i}\right)=\emptyset
$$

i.e., the economy $\mathcal{E}(\boldsymbol{v})$ satisfies Assumption (NS).

Applying Theorem 3.1 to the economy $\mathcal{E}(\boldsymbol{v})$ there exists a quasi-equilibrium $(p, \boldsymbol{x})$ of $\mathcal{E}(\boldsymbol{v})$. Therefore the allocation $\boldsymbol{x}$ is feasible and for each $i$ 
(a) the action $x^{i}$ satisfies the budget restriction $p \cdot x^{i} \leqslant p \cdot e^{i}$;

(b) the action $x^{i}$ is weakly optimal for the utility function $v^{i}$ in the budget set, i.e., for each $y^{i} \in X^{i}$,

$$
v^{i}\left(y^{i}\right)>v^{i}\left(x^{i}\right) \Longrightarrow p \cdot y^{i} \geqslant p \cdot e^{i} .
$$

We claim that $(p, \boldsymbol{x})$ is a quasi-equilibrium of the economy $\mathcal{E}(\boldsymbol{u})$. To see this it is sufficient to prove that $x^{i}$ is weakly optimal for the utility function $u^{i}$ in the budget set. Let $y^{i} \in X^{i}$, if $u^{i}\left(y^{i}\right)>u^{i}\left(x^{i}\right)$ then $x^{i}$ does not belong to $S^{i}\left(u^{i}\right)$ and $u^{i}\left(x^{i}\right)=v^{i}\left(x^{i}\right)$. Since $v^{i}\left(y^{i}\right) \geqslant u^{i}\left(y^{i}\right)$, we obtain that $v^{i}\left(y^{i}\right)>v^{i}\left(x^{i}\right)$, implying from (6.8) that $p \cdot y^{i} \geqslant p \cdot e^{i}$.

\subsection{Economies with two agents}

If there are two agents then Conjecture 4.1 is valid.

Proposition 6.1. If there are at most two agents, then the strong compactness of the individually rational utility set follows from its compactness.

Proof of Proposition 6.1 Let $\mathcal{E}(\boldsymbol{u})$ be an economy with two agents, ${ }^{9}$ i.e., $I=$ $\left\{i_{1}, i_{2}\right\}$. Assume that the individually rational utility set is compact. We have to prove that Assumption (SCU) is satisfied. Let $\left(\boldsymbol{x}_{n}\right)_{n}$ be a sequence in $\mathrm{F} \cap \operatorname{Ir}(\boldsymbol{u})$. Since the individually rational utility set is compact, there exists a subsequence $\left(\boldsymbol{x}_{n_{k}}\right)_{k}$ satisfying

$$
\forall i \in I, \quad u^{i}\left(y^{i}\right) \geqslant \lim _{k \rightarrow \infty} u^{i}\left(x_{n_{k}}^{i}\right) .
$$

If the sequence $\left(x_{n_{k}}^{i_{1}}\right)_{k}$ is bounded then, by feasibility, the sequence $\left(x_{n_{k}}^{i_{2}}\right)_{k}$ is also bounded. Passing to a subsequence if necessary, there exists $\boldsymbol{x}$ such that

$$
\forall i \in I, \quad \lim _{k \rightarrow \infty}\left(x^{i}-x_{n_{k}}^{i}\right)=0 .
$$

Since utility functions are upper semi-continuous, passing to a subsequence if necessary we have

$$
\forall i \in I, \quad \lim _{k \rightarrow \infty} u^{i}\left(x_{n_{k}}^{i}\right) \leqslant u^{i}\left(x^{i}\right) .
$$

Assumption (SCU) follows from the fact that for each $i$ the sequence $\left(1+\left\|x_{n_{k}}^{i}\right\|\right)_{k}$ is bounded.

Assume now that the the sequence $\left(x_{n_{k}}^{i_{1}}\right)_{k}$ is not bounded. By feasibility, it follows that the sequence $\left(x_{n_{k}}^{i_{2}}\right)_{k}$ is also unbounded. Passing to a subsequence if necessary, we may assume that

$$
\forall i \in I, \quad \lim _{k \rightarrow \infty}\left\|x_{n_{k}}^{i}\right\|=+\infty .
$$

\footnotetext{
${ }^{9}$ We do not consider the trivial case of an economy with only one agent.
} 
But since

$$
\lim _{k \rightarrow \infty} \frac{1}{\left\|x_{n_{k}}^{i}\right\|^{2}}\left\|y^{i}-x_{n_{k}}^{i}\right\|=\lim _{k \rightarrow \infty} \frac{\left\|x_{n_{k}}^{i}\right\|}{\left\|x_{n_{k}}^{i}\right\|^{2}}=\lim _{k \rightarrow \infty} \frac{1}{\left\|x_{n_{k}}^{i}\right\|}=0
$$

Assumption (SCU) is satisfied.

As a direct consequence of Theorem 6.1 we obtain the following existence result.

Corollary 6.1. Consider an economy with at most two agents. If

(a.1) the individually rational utility $\operatorname{set} \mathcal{U}(\boldsymbol{u})$ is compact,

(b.1) the weak non-satiation condition (WNS) is satisfied,

then there exists a quasi-equilibrium.

\subsection{Compactness of feasible and individually rational allocations}

We propose to prove that our main existence result Theorem 6.1 generalizes Theorem 2 in Allouch and Le Van (2008). If the set $\mathrm{F} \cap \operatorname{Ir}(\boldsymbol{u})$ of feasible and individually rational allocations is compact then every standard economy is strongly standard.

Proposition 6.2. If the set $\mathrm{F} \cap \operatorname{Ir}(\boldsymbol{u})$ of feasible and individually rational allocations is compact then the individually rational utility set $\mathcal{U}(\boldsymbol{u})$ is strongly compact.

Proof of Proposition 6.2 Let $\mathcal{E}(\boldsymbol{u})$ be an economy such that the set $\mathrm{F} \cap \operatorname{Ir}(\boldsymbol{u})$ is compact. We have to prove that Assumption (SCU) is satisfied. Let $\left(\boldsymbol{x}_{n}\right)_{n}$ be a sequence in $\mathrm{F} \cap \operatorname{Ir}(\boldsymbol{u})$. By compactness, there exist a subsequence $\left(\boldsymbol{x}_{n_{k}}\right)_{k}$ and an allocation $x$ satisfying

$$
\forall i \in I, \quad \lim _{k \rightarrow \infty}\left(x^{i}-x_{n_{k}}^{i}\right)=0
$$

Since utility functions are upper semi-continuous, passing to a subsequence if necessary we have

$$
\forall i \in I, \quad \lim _{k \rightarrow \infty} u^{i}\left(x_{n_{k}}^{i}\right) \leqslant u^{i}\left(x^{i}\right) .
$$

Assumption (SCU) follows from the fact that for each $i$ the sequence $\left(1+\left\|x_{n_{k}}^{i}\right\|\right)_{k}$ is bounded.

As a direct consequence of Theorem 6.1 we obtain the main existence result in Allouch and Le Van (2008).

Corollary 6.2. If the two following conditions are satisfied 
(a.3) the set $\mathrm{A}(\boldsymbol{u})=\mathrm{F} \cap \operatorname{Ir}(\boldsymbol{u})$ of feasible and individually rational allocations is compact,

(b.1) the the weak non-satiation condition (WNS) is satisfied, then there exists a quasi-equilibrium.

\subsection{Economies satisfying a no-arbitrage condition}

In order to introduce a no-arbitrage condition, we recall some definitions. For each action $x^{i} \in X^{i}$, we denote by $\mathrm{As}^{i}\left(x^{i}\right)$ the asymptotic (or recession) cone of the set $\widehat{P}^{i}\left(x^{i}\right)=\left\{y^{i} \in X^{i}: u^{i}\left(y^{i}\right) \geqslant u^{i}\left(x^{i}\right)\right\}$, i.e.

$$
\operatorname{As}^{i}\left(x^{i}\right):=\left\{v \in \mathbb{R}^{J}: \forall t \geqslant 0, \forall y^{i} \in \widehat{P}^{i}\left(x^{i}\right), y^{i}+t v \in \widehat{P}^{i}\left(x^{i}\right)\right\} .
$$

We denote by $\mathrm{L}^{i}\left(x^{i}\right)$ the lineality space defined by

$$
\mathrm{L}^{i}\left(x^{i}\right):=\operatorname{As}^{i}\left(x^{i}\right) \cap-\operatorname{As}^{i}\left(x^{i}\right) .
$$

Let $\operatorname{Span}\left(X^{i}\right)$ the smallest linear subspace of $\mathbb{R}^{J}$ containing $X^{i}$. The lineality space $\mathrm{L}^{i}\left(x^{i}\right)$ is the largest linear subspace of $\operatorname{Span}\left(X^{i}\right)$ contained in the $\operatorname{As}^{i}\left(x^{i}\right)$. We borrow from Allouch et al. (2006) the following concept of no-arbitrage condition.

Definition 6.2. An economy satisfying the two following conditions

(a) for each agent $i$ and for individually rational allocation $x^{i} \in \widehat{P}^{i}\left(e^{i}\right)$ we have

$$
\mathrm{L}^{i}\left(x^{i}\right)=\mathrm{L}^{i}\left(e^{i}\right)
$$

(b) for every family $\left(y^{i}\right)_{i \in I}$ with $y^{i} \in \operatorname{As}^{i}\left(e^{i}\right)$,

$$
\sum_{i \in I} y^{i}=0 \Longrightarrow y^{i} \in \mathrm{L}^{i}\left(e^{i}\right), \quad \forall i \in I
$$

is called a no-arbitrage economy.

In Allouch et al. (2006) condition (a) is called weak uniformity and condition (b) is called weak no market arbitrage.

Proposition 6.3. For every no-arbitrage economy, the strong compactness of the individually rational utility set follows from its compactness.

Proof of Proposition 6.3 To begin, let $\left[\mathrm{L}^{i}\left(e^{i}\right)\right]^{\perp}$ be the subspace of $\operatorname{Span}\left(X^{i}\right)$ orthogona ${ }^{10}$ to $\mathrm{L}\left(e^{i}\right)$. Every vector $x^{i} \in X^{i}$ can be uniquely decomposed on a sum $\Psi^{i}\left(x^{i}\right)+\Theta^{i}\left(x^{i}\right)$ where $\Psi^{i}\left(x^{i}\right) \in\left[\mathrm{L}^{i}\left(e^{i}\right)\right]^{\perp}$ and $\Theta^{i}\left(x^{i}\right) \in \mathrm{L}^{i}\left(e^{i}\right)$.

Consider now a no-arbitrage economy such that the individually rational utility set is compact. Let $\left(\boldsymbol{x}_{n}\right)_{n}$ be a sequence of feasible and individually rational allocations. Passing to a subsequence if necessary we can assume that $I=I_{c} \cup I_{u}$ where

\footnotetext{
${ }^{10}$ For the usual scalar product in $\mathbb{R}^{J}$.
} 
(a) for each $i \in I_{c}$, there exists $x^{i} \in X^{i}$ such that the sequence $\left(x_{n}^{i}\right)_{n}$ converges to $x^{i}$,

(b) for each $i \in I_{u}$, the sequence $\left(\left\|x_{n}^{i}\right\|\right)_{n}$ converges to $+\infty$.

For each $i \in I_{u}$, we let $z_{n}^{i}:=\Psi^{i}\left(x_{n}^{i}\right)$.

Claim 6.3. For each $i \in I_{u}$, the sequence $\left(z_{n}^{i}\right)_{n}$ is bounded.

Proof of Claim 6.3. Let $\alpha_{n}=\max \left\{\left\|z_{n}^{i}\right\|: i \in I_{u}\right\}$ and assume by way of contradiction that the sequence $\left(\alpha_{n}\right)_{n}$ is not bounded. Passing to a subsequence if necessary, we can assume that $\lim _{n \rightarrow \infty} \alpha_{n}=+\infty$ and there exists $\left(\xi^{i}\right)_{i \in I_{u}} \neq 0$ such that

$$
\forall i \in I_{u}, \quad \lim _{n \rightarrow \infty} \frac{1}{\alpha_{n}} z_{n}^{i}=\zeta^{i} .
$$

Since $\boldsymbol{x}_{n}$ is feasible we have

$$
\sum_{i \in I_{c}} x_{n}^{i}+\sum_{i \in I_{u}} x_{n}^{i}=\sum_{i \in I} e^{i} .
$$

Dividing by $\alpha_{n}$ and passing to the limit, we obtain

$$
\sum_{i \in I_{u}} \zeta^{i}=0
$$

Recall that $z_{n}^{i}=x_{n}^{i}-\Theta^{i}\left(x_{n}^{i}\right)$ with $\Theta^{i}\left(x_{n}^{i}\right) \in \mathrm{L}^{i}\left(e^{i}\right)$. Since $x_{n}^{i} \in \widehat{P}^{i}\left(e^{i}\right)$ it follows that $z_{n}^{i}$ belongs to $\widehat{P}^{i}\left(e^{i}\right)$. Following Exercice 1.17 in Florenzano and Le Van (2001), we obtain that $\zeta^{i}$ belongs to $\operatorname{As}^{i}\left(e^{i}\right)$. The economy satisfies the weak no market arbitrage condition. It follows that $\zeta^{i} \in \mathrm{L}^{i}\left(e^{i}\right)$ for each $i \in I_{u}$. But $\zeta^{i}$ also belongs to $\left[\mathrm{L}^{i}\left(e^{i}\right)\right]^{\perp}$, implying that $\zeta^{i}=0$ for each $i \in I_{u}$ : contradiction.

Passing to a subsequence if necessary we can assume that for each $i \in I_{u}$, there exists $z^{i} \in X^{i}$ such that the sequence $\left(z_{n}^{i}\right)_{n}$ converges to $z^{i}$. For each $n$ we have

$$
\sum_{i \in I_{c}} x_{n}^{i}+\sum_{i \in I_{u}} z_{n}^{i}-\sum_{i \in I} e^{i} \in \sum_{i \in I_{u}} \mathrm{~L}^{i}\left(e^{i}\right) .
$$

Therefore there exists $\left(\eta^{i}\right)_{i \in I_{u}}$ such that $\eta^{i} \in \mathrm{L}^{i}\left(e^{i}\right)$ and

$$
\sum_{i \in I_{c}} x^{i}+\sum_{i \in I_{u}} z^{i}-\sum_{i \in I} e^{i}=\sum_{i \in I_{u}} \eta^{i}
$$

For each $i \in I_{u}$ we let $y^{i}:=z^{i}-\eta^{i}$ and for each $i \in I_{c}$ we let $y^{i}=x^{i}$. Since $z_{n}^{i} \in \widehat{P}^{i}\left(e^{i}\right)$ for each $n$ it follows that $z^{i} \in \widehat{P}^{i}\left(e^{i}\right)$. Since $\eta^{i} \in \mathrm{L}^{i}\left(e^{i}\right)$ it follows that $y^{i} \in \widehat{P}^{i}\left(e^{i}\right)$. Therefore the allocation $\boldsymbol{y}=\left(y_{i}\right)_{i \in I}$ is feasible and individually rational, i.e., $\boldsymbol{y} \in \mathrm{F} \cap \operatorname{Ir}(\boldsymbol{u})$. 
Since $u^{i}$ is upper semi-continuous, passing to a subsequence if necessary we have

$$
\forall i \in I_{c}, \quad u^{i}\left(y^{i}\right) \geqslant \lim _{n \rightarrow \infty} u^{i}\left(x_{n}^{i}\right)
$$

and

$$
\forall i \in I_{u}, \quad u^{i}\left(z^{i}\right) \geqslant \lim _{n \rightarrow \infty} u^{i}\left(z_{n}^{i}\right) .
$$

Recall that $z_{n}^{i}=x_{n}^{i}-\Theta^{i}\left(x_{n}^{i}\right)$ with $\Theta^{i}\left(x_{n}^{i}\right) \in \mathrm{L}^{i}\left(e^{i}\right)$. The economy satisfies the weak uniformity condition. This implies that $\Theta^{i}\left(x_{n}^{i}\right) \in \mathrm{L}^{i}\left(x_{n}^{i}\right)$ and $z_{n}^{i} \in \widehat{P}^{i}\left(x_{n}^{i}\right)$. Therefore

$$
\forall i \in I_{u}, \quad u^{i}\left(z^{i}\right) \geqslant \lim _{n \rightarrow \infty} u^{i}\left(z_{n}^{i}\right) \geqslant \lim _{n \rightarrow \infty} u^{i}\left(x_{n}^{i}\right) .
$$

In order to prove that the economy is strongly standard it is sufficient to prove that

$$
\forall i \in I, \quad \lim _{n \rightarrow \infty} \frac{1}{\left\|x_{n}^{i}\right\|^{2}}\left(y^{i}-x_{n}^{i}\right)=0 .
$$

If $i \in I_{c}$ then

$$
\lim _{n \rightarrow \infty} \frac{1}{\left\|x_{n}^{i}\right\|^{2}}\left(y^{i}-x_{n}^{i}\right)=\frac{1}{\left\|y^{i}\right\|^{2}} \lim _{n \rightarrow \infty}\left(y^{i}-x_{n}^{i}\right)=0 .
$$

Observe that for each $i$ there exists $M^{i}>0$ such that

$$
\forall n \in \mathbb{N}, \quad \frac{1}{\left\|x_{n}^{i}\right\|}\left\|y^{i}-x_{n}^{i}\right\| \leqslant M^{i} .
$$

Passing to a subsequence if necessary we may assume that the sequence $\left(\left(y^{i}-\right.\right.$ $\left.\left.x_{n}^{i}\right) /\left\|x_{n}^{i}\right\|\right)_{n}$ converges. Now fix $i \in I_{u}$, we have

$$
\lim _{n \rightarrow \infty} \frac{1}{\left\|x_{n}^{i}\right\|^{2}}\left(y^{i}-x_{n}^{i}\right)=\lim _{n \rightarrow \infty} \frac{1}{\left\|x_{n}^{i}\right\|} \lim _{n \rightarrow \infty} \frac{1}{\left\|x_{n}^{i}\right\|}\left(y^{i}-x_{n}^{i}\right)=0 .
$$

As a direct consequence of Theorem 6.1 we obtain the following existence result.

Corollary 6.3. Consider a no-arbitrage economy. If the following conditions are satisfied

(a.1) the individually rational utility $\operatorname{set} \mathcal{U}(\boldsymbol{u})$ is compact,

(b.1) the weak non-satiation condition (WNS) is satisfied,

then there exists a quasi-equilibrium.

Recently, Allouch et al. (2006) proved that every no-arbitrage economy with a compact individually rational utility set, admits a quasi-equilibrium provided that the following non-satiation condition is satisfied: 
(ANS) For every $x^{i} \in A^{i}(\boldsymbol{u})$, if $x^{i} \in S^{i}\left(u^{i}\right)$ then $\operatorname{As}^{i}\left(x^{i}\right) \backslash \mathrm{L}^{i}\left(x^{i}\right) \neq \emptyset$.

Actually, for no-arbitrage economies, the non-satiation condition (ANS) implies the weak non-satiation condition (WNS).

Proposition 6.4. Every no-arbitrage economy satisfying Assumption (ANS) actually satisfies Assumption (WNS).

As a consequence of Proposition 6.4, the existence result in Allouch et al. (2006) is a particular case of Corollary 6.3.

Proof of Proposition 6.4 Consider a no-arbitrage economy satisfying Assumption (ANS). Fix an agent $k \in I$ and an action $x^{k} \in A^{k}(\boldsymbol{u})$. Since Assumption (ANS) is satisfied we can choose a vector $\xi^{k} \in \mathrm{As}^{k}\left(x^{k}\right) \backslash \mathrm{L}^{k}\left(x^{k}\right)$. In order to prove that Assumption (WNS) is satisfied, it is sufficient to prove that for some $t>0$ the vector $x^{i}+t \xi^{i}$ does not belong to $A^{i}(\boldsymbol{u})$. Assume by way of contradiction that for every $n \in \mathbb{N}$ the vector $x^{k}+t_{n} \xi^{k}$ belongs to $A^{k}(\boldsymbol{u})$ where $t_{n}=n+1$. For each $n$, there exist a feasible and individually rational allocation $\boldsymbol{y}_{n}$ in $\mathrm{F} \cap \operatorname{Ir}(\boldsymbol{u})$ such that $y_{n}^{k}=x^{k}+t_{n} \xi^{k}$. In particular we have

$$
\forall n \in \mathbb{N}, \quad x^{k}+t_{n} \xi^{k}+\sum_{i \in I \backslash\{k\}} \Psi^{i}\left(y_{n}^{i}\right)+z_{n}=\sum_{i \in I} e^{i}
$$

where $z_{n}$ is the vector in $\sum_{i \in I} \mathrm{~L}^{i}\left(e^{i}\right)$ defined by

$$
e_{n}:=\sum_{i \in I \backslash\{k\}} \Theta^{i}\left(y_{n}^{i}\right) .
$$

For each $n$ we let

$$
\alpha_{n}:=\max \left\{t_{n}\left\|\xi^{k}\right\|,\left\|z_{n}\right\|, \max _{i \neq k}\left\{\left\|\Psi^{i}\left(y_{n}^{i}\right)\right\|\right\}\right\} .
$$

Since $\left(t_{n}\right)_{n}$ converges to $+\infty$, the sequence $\left(\alpha_{n}\right)_{n}$ also converges to $+\infty{ }^{11}$ and passing to the limit in 6.9 ) we have

$$
\sum_{i \in I} \zeta^{i}+\eta=0
$$

where (passing to a subsequence if necessary)

$$
\zeta^{k}=\lim _{n \rightarrow \infty} \frac{t_{n}}{\alpha_{n}} \xi^{k}, \quad \zeta^{i}=\lim _{n \rightarrow \infty} \frac{1}{\alpha_{n}} \Psi^{i}\left(y_{n}^{i}\right) \quad \text { and } \quad \eta=\lim _{n \rightarrow \infty} \frac{1}{\alpha_{n}} z_{n} .
$$

By construction of $\alpha_{n}$, we have that $\zeta=\left(\zeta^{i}\right)_{i \in I} \neq 0$ or $\eta \neq 0$.

Claim 6.4. For each $i \in I$, we have $\zeta^{i} \in \operatorname{As}^{i}\left(e^{i}\right)$ and $\eta \in \sum_{i \in I} \mathrm{~L}^{i}\left(e^{i}\right)$.

\footnotetext{
${ }^{11}$ Since $\xi^{k}$ does not belong to $\mathrm{L}^{k}\left(e^{k}\right)$ we cannot have $\xi^{k}=0$..
} 
Proof of Claim 6.4 Fix $i \in I$ with $i \neq k$. We know that $u^{i}\left(y_{n}^{i}\right) \geqslant u^{i}\left(e^{i}\right)$. Since $\Theta^{i}\left(y_{n}^{i}\right)$ belongs to $\mathrm{L}^{i}\left(e^{i}\right)$ we have

$$
u^{i}\left(\Psi^{i}\left(y_{n}^{i}\right)\right)=u^{i}\left(y_{n}^{i}-\Theta^{i}\left(y_{n}^{i}\right)\right) \geqslant u^{i}\left(y_{n}^{i}\right) \geqslant u^{i}\left(e^{i}\right),
$$

implying that $\zeta^{i} \in \mathrm{As}^{i}\left(e^{i}\right)$. The relation $\eta \in \sum_{i \in I} \mathrm{~L}^{i}\left(e^{i}\right)$ comes from the fact that the subspace $\sum_{i \in I} \mathrm{~L}^{i}\left(e^{i}\right)$ is closed.

There exists a family $\left(\eta^{i}\right)_{i \in I}$ with $\eta^{i} \in \mathrm{L}^{i}\left(e^{i}\right)$ such that $\eta=\sum_{i \in I} \eta^{i}$. It then follows from 6.10 , that

$$
\sum_{i \in I}\left(\zeta^{i}+\eta^{i}\right)=0
$$

Since the economy satisfies the weak no market arbitrage condition, we have $\zeta^{i}+\eta^{i} \in \mathrm{L}^{i}\left(e^{i}\right)$. Recall that $\zeta^{i} \in\left[\mathrm{L}\left(e^{i}\right)\right]^{\perp}$, implying that $\zeta^{i}=0$ for each $i$ and $\eta=0$ : contradiction.

\section{A An alternative proof of Theorem 2 in Allouch and Le Van (2007)}

Allouch and Le Van (2008) proved that if

(a.3) the set $\mathrm{A}(\boldsymbol{u})=\mathrm{F} \cap \operatorname{Ir}(\boldsymbol{u})$ of feasible and individually rational allocations is compact,

(b.1) the the weak non-satiation condition (WNS) is satisfied,

then there exists a quasi-equilibrium. In this section we propose an alternative proof of this result.

Proof of Theorem 4.1 Let $\mathcal{E}(\boldsymbol{u})=\left(X^{i}, u^{i}, e^{i}\right)_{i \in I}$ be an economy with a compact set $\mathrm{A}(\boldsymbol{u})$ of feasible and individually rational allocations and satisfying the weak non-satiation Assumption (WNS). We split the set of agents in two parts: we let $I_{n s}=\left\{i \in I: S^{i}\left(u^{i}\right)=\emptyset\right\}$ be the set of agents that are never satiated and we let $I_{s}=\left\{i \in I: S^{i}\left(u^{i}\right) \neq \emptyset\right\}$ be the set of agents that may be satiated. We propose to modify the characteristics of the agents that may be satiated. Fix $i \in I_{s}$. The set $\mathrm{A}^{i}(\boldsymbol{u})$ is compact and since $u^{i}$ is upper semi-continuous, there exists $\xi^{i} \in \operatorname{argmax}\left\{u^{i}(x): x \in \mathrm{A}^{i}(\boldsymbol{u})\right\}$. Applying Assumption (WNS),

$$
\exists \zeta^{i} \in S^{i}\left(u^{i}\right) \backslash A^{i}(\boldsymbol{u}), \quad u^{i}\left(\zeta^{i}\right) \geqslant u^{i}\left(\xi^{i}\right)=\sup \left\{u^{i}(x): x \in \mathrm{A}^{i}(\boldsymbol{u})\right\} .
$$

We consider another economy $\mathcal{G}=\left(Y^{i}, Q^{i}, e^{i}\right)_{i \in I}$ with non-ordered preferences and such that for each $i$ the consumption set $Y^{i}$ is defined by

$$
Y^{i}=\left\{x^{i} \in X^{i}: u^{i}\left(x^{i}\right) \geqslant u^{i}\left(e^{i}\right)\right\}
$$


and for each bundle $y^{i} \in Y^{i}$ the set $Q^{i}\left(y^{i}\right)$ of strictly preferred bundles is defined by ${ }^{12}$

$$
Q^{i}\left(y^{i}\right)= \begin{cases}P^{i}\left(y^{i}\right) \cup\left\{\zeta^{i}\right\} & \text { if } \quad i \in I_{s} \\ P^{i}\left(y^{i}\right) & \text { if } i \in I_{n s}\end{cases}
$$

where $P^{i}\left(y^{i}\right)=\left\{z^{i} \in X^{i}: u^{i}\left(z^{i}\right)>u^{i}\left(y^{i}\right)\right\}$.

Applying Assumptions (A.1) and (A.2), the consumption set $Y^{i}$ is closed convex and contains the initial endowment $e^{i}$. Observe that the set of feasible allocations of the economy $\mathcal{G}$ coincides with the set $\mathrm{A}(\boldsymbol{u})$ of individually rational and feasible allocations of the economy $\mathcal{E}(\boldsymbol{u})$. In particular it is compact. By construction, for each feasible allocation $\boldsymbol{y}=\left(y^{i}\right)_{i \in I}$ the strictly preferred set $Q^{i}\left(y^{i}\right)$ is non-empty. Moreover, since $\zeta^{i} \notin \mathrm{A}^{i}(\boldsymbol{u})$ we have $y^{i} \notin Q^{i}\left(y^{i}\right)$ for each individually feasible bundle $y^{i}$. In order to apply Proposition 3.2.3 in Florenzano (2003) it is sufficient to prove the following claim.

Claim A.1. For each $i$ the correspondence $Q^{i}$ has convex upper sections ${ }^{13}$ and open lower sections. ${ }^{14}$

Proof of Claim A.1. The claim is obvious if $i \in I_{n s}$. Let $i \in I_{s}$. For each bundle $y^{i} \in Y^{i}$, we have

$$
Q^{i}\left(y^{i}\right)=\left\{\begin{array}{lll}
P^{i}\left(y^{i}\right) & \text { if } & y^{i} \notin S^{i} \\
\left\{\zeta^{i}\right\} & \text { if } & y^{i} \in S^{i}
\end{array}\right.
$$

It follows that $Q^{i}$ has convex values. For each $z^{i} \in Y^{i}$, we have

$$
\left(Q^{i}\right)^{-1}\left(z^{i}\right)= \begin{cases}\left(P^{i}\right)^{-1}\left(z^{i}\right) \cap Y^{i} & \text { if } z^{i} \neq \zeta^{i} \\ Y^{i} & \text { if } z^{i}=\zeta^{i} .\end{cases}
$$

It follows that $Q^{i}$ has open lower sections.

We can now apply Proposition 3.2.3 in Florenzano (2003) to obtain the existence of a quasi-equilibrium of $\mathcal{G}$ which is obviously a quasi-equilibrium of $\mathcal{E}(\boldsymbol{u})$.

\section{References}

Allingham, M.: 1991, Existence theorems in the capital asset pricing model, Econometrica 59(4), 1169-1174.

Allouch, N.: 2002, An equilibrium existence result with short selling, Journal of Mathematical Economics 37(2), 81-94.

\footnotetext{
${ }^{12}$ Observed that $\zeta^{i} \in Y^{i}$ for each $i$.

${ }^{13}$ I.e. for each bundle $y^{i} \in Y^{i}$, the set $Q^{i}\left(y^{i}\right)$ is convex.

${ }^{14}$ I.e. for each $z^{i} \in Y^{i}$, the set $\left(Q^{i}\right)^{-1}\left(z^{i}\right)=\left\{y^{i} \in Y^{i}: Q^{i}\left(y^{i}\right) \ni z^{i}\right\}$ is open in $Y^{i}$.
} 
Allouch, N. and Le Van, C.: 2008, Walras and dividends equilibrium with possibly satiated consumers. Queen Mary University of London, Working Paper \# 555 .

Allouch, N., Le Van, C. and Page, Jr., F. H.: 2002, The geometry of arbitrage and the existence of competitive equilibrium, Journal of Mathematical Economics 38(4), 373-391.

Allouch, N., Le Van, C. and Page, Jr., F. H.: 2006, Arbitrage and equilibrium in unbounded exchange economies with satiation, Journal of Mathematical Economics 42(6), 661-674.

Arrow, K. J. and Debreu, G.: 1954, Existence of an equilibrium for a competitive economy, Econometrica 22, 265-290.

Dana, R.-A., Le Van, C. and Magnien, F.: 1999, On the different notions of arbitrage and existence of equilibrium, Journal of Economic Theory 87(1), 169193.

Florenzano, M.: 2003, General Equilibrium Analysis: Existence and Optimality Properties of Equilibria, Kluwer Academic Publishers.

Florenzano, M. and Le Van, C.: 2001, Finite dimensional convexity and optimization, Vol. 13 of Studies in Economic Theory, Springer-Verlag, Berlin. In cooperation with Pascal Gourdel.

Hammond, P. J.: 1983, Overlapping expectations and Hart's condition for equilibrium in a securities model, Journal of Economic Theory 31(1), 170-175.

Hart, O. D.: 1974, On the existence of equilibrium in a securities model, Journal of Economic Theory 9(3), 293-311.

Le Van, C., Page, Jr., F. H. and Wooders, M. H.: 2001, Arbitrage and equilibrium in economies with externalities, Journal of Global Optimization 20(34), 309-321.

McKenzie, L. W.: 1959, On the existence of general equilibrium for a competitive market, Econometrica 27, 54-71.

Nielsen, L. T.: 1989, Asset market equilibrium with short-selling, Review of Economic Studies 56(3), 467-473.

Page, Jr., F. H.: 1987, On equilibrium in Hart's securities exchange model, Journal of Economic Theory 41(2), 392-404.

Page, Jr., F. H. and Wooders, M. H.: 1996, A necessary and sufficient condition for the compactness of individually rational and feasible outcomes and the existence of an equilibrium, Economics Letters 52(2), 153-162. 
Page, Jr., F. H., Wooders, M. H. and Monteiro, P. K.: 2000, Inconsequential arbitrage, Journal of Mathematical Economics 34(4), 439-469.

Werner, J.: 1987, Arbitrage and the existence of competitive equilibrium, Econometrica 55(6), 1403-1418.

Won, D., Hahn, G. and Yannelis, N.: 2008, Capital market equilibrium without riskless assets: heterogeneous expectations, Annals of Finance 4(2), 183195. 